\title{
Energy Efficiency Optimisation in Heat Treatment Process Design
}

\author{
Iñigo Mendikoa ${ }^{1}$, Mikel Sorli ${ }^{1}$, Alberto Armijo ${ }^{1}$, Laura Garcia ${ }^{1}$, Luis Erausquin ${ }^{2}$, \\ Mario Insunza ${ }^{3}$, Jon Bilbao ${ }^{3}$, Hakan Friden ${ }^{4}$, Anders Björk ${ }^{4}$, Linus Bergfors ${ }^{4}$, \\ Romualdas Skema ${ }^{5}$, Robertas Alzbutas ${ }^{5}$, and Tomas Iesmantas ${ }^{5}$ \\ ${ }^{1}$ Tecnalia Research\&Innovation \\ \{inigo.mendikoa, mikel.sorli, alberto.armijo, \\ laura.garciazambrano\} @tecnalia.com \\ ${ }^{2}$ Fundiciones del Estanda \\ lerausquin@estanda.com \\ ${ }^{3}$ Sisteplant S.L. \\ \{minsunza, jbilbao\} @sisteplant.com \\ ${ }^{4}$ IVL Swedish Environmental Institute Ltd. \\ \{hakan.friden, anders.bjork, linus.bergfors\}@ivl.se \\ ${ }^{5}$ Lietuvos Energetikos Institutas \\ \{skema, robertas, iesmantas\} @mail. lei.lt
}

\begin{abstract}
Information and Communication Technology (ICT) tools to support design in terms of energy efficiency optimisation are beginning to come into the market, in particular for energy savings and management in buildings, however little has yet been developed for manufacturing. The work here described is related to the enhancement of existing manufacturing process design methodology and tools with a set of ICT components developed allowing process knowledge based design, simulation and optimisation in terms of energy efficiency. In particular the work described is focused on the heat treatment process of steel casting parts. This process design traditionally consists of a suitable predefined temperature-time curve selection based on personal experience and given customer requirements (material and mechanical properties), yet this curve can actually be optimised in terms of energy consumption and maintenance cost, while keeping required mechanical properties.
\end{abstract}

Keywords: Energy efficiency, Process design optimisation, Heat treatment.

\section{Introduction}

There are several ICT tools to support process design, addressing especially installation customization to the users' needs. Many of them include simulation of processes, which might be used to implicitly analyse energy use within the design processes. Research and technology development attempts to address sustainability issues in process design have been made and several tools are available on the market intending to support users in consideration of sustainability problems. However, a 
systematic analysis of energy use within the designed processes and functionality to explicitly support the designer in making decisions relevant for energy efficiency are not provided in most of the currently available design and simulation tools. The current design tools do not explicitly support decisions regarding selection of methods, equipment and materials to use for meeting specifications and for energy efficiency over the whole process life cycle, especially in flexible manufacturing systems asking often for reconfiguration of installations. ICT tools to support design for energy efficiency optimisation are beginning to come into the market, in particular for energy savings and management in buildings, however little has yet been developed for manufacturing.

The work here described is related to the enhancement of existing manufacturing process design methodology and tools with a set of ICT components developed in the framework of DEMI project (FP7-ICT-2009-247831). In particular the work here described is focused on the heat treatment process of steel casting parts and is related to the company Fundiciones del Estanda in Spain, allowing this company to address energy efficiency in this process design.

\section{Manufacturing Process and Energy Consumption Characteristics}

Manufacturing process at this company is related to steel casting parts including subprocesses like melting, machining, grinding, cutting and cleaning and heat treatment. This heat treatment process is powered by gas energy, and considering that over $33 \%$ of total gas at the company is consumed here, it is reasonable to focus the energy efficient design on this specific sub-process. The main goal is therefore to reduce the energy consumption on the heat treatment process while at least keeping the productivity and resulting mechanical requirements in the steel parts, as well as maintenance costs acceptable.

The specific thermal process design for each piece begins with the selection of the suitable temperature-time curve for the part to be treated, which is defined based on personal experience of experts in charge of process design. The "process design" basically consists of the definition of the Temperature-Time curve for the heat treatment for each type of part, given customer requirements (material and mechanical properties). Predefined Temperature-time curves are normally used, yet these curves can actually be optimised in terms of energy consumption, however this optimisation is currently not done at the company. The DEMI tool being developed is intended to provide the company with the capability of designing optimal heat treatment processes.

Heat treatment process, described by the $T$ - $t$ curve, usually consists of some few steps, which can be simplified as an initial heating, temperature holding time $t$, and a final cooling, but it might be more complex. The considered case study will focus on a particular steel part: brake discs for the high-speed train treated in the Guinea 4 batch furnace. DEMI system will propose the optimal $T$ - $t$ curve, consuming minimum gas providing the mechanical requirements, while maintenance costs must remain acceptable so that the new process is profitable. The software can also optimise the process design in terms of minimal maintenance cost, or even use a multicriteria optimisation strategy considering both weighted energy and maintenance cost. 


\section{Process Optimisation with DEMI Tools}

DEMI ICT tool consists of several ICT components, three of which are involved in the work being implemented at the manufacturing company, namely, 'Energy Dependency Selector' (EDS) supporting a knowledge based preliminary process design, 'Energy Analyser' (EA) for process optimisation and the supporting 'Energy Simulator' (ES) for energy consumption estimation in specific process configuration. Fig. 1 shows the generic process design procedure based on DEMI tools (in grey). All DEMI components communicate each other via web services and sharing information in a Knowledge Repository (KR). In earlier paper the connection between KR-EA-ES for optimisation of configuration of compressed air systems has been described [1].

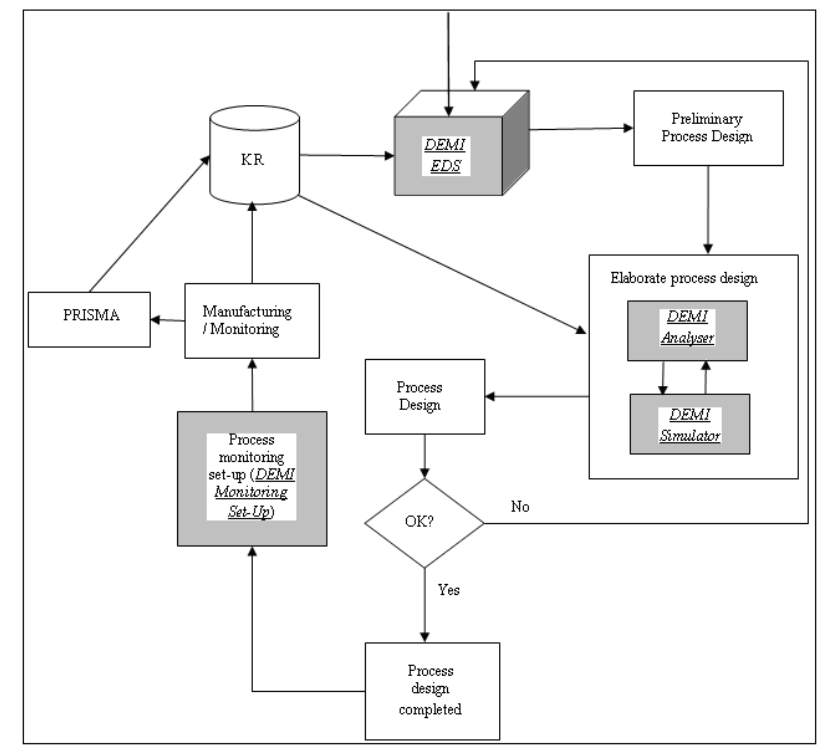

Fig. 1. Manufacturing process optimal design with DEMI components

\subsection{Knowledge Based Preliminary Process Design}

Preliminary process design can be quickly defined based on experts' experience, described in terms of rules and successful historic processes. This knowledge based preliminary process design is done with the support of the DEMI component Energy Dependency Selector (EDS), allowing the process designer to quickly figure out the consequences, also in terms of energy consumption, of decisions at process early design stage. EDS component provides a preliminary process design based on two techniques: Case Based Reasoning (CBR) and Rule Based Reasoning (RBR).

Case Based Reasoning. This functionality provides historic process designs 'similar' to the target process requirements given by the designer. In this case the requirements are those from the customer, basically material and mechanical properties required. 
This functionality requires historic cases database defined and populated including parameters describing the requirements, in addition to those describing the process design. The 'similarity' of historic processes with respect to required parameters values provided by the user is estimated according to the relative weights given for each parameter. The tool finally provides a list of historic processes sorted by similarity. This functionality implementation is based on the JColibri tool $[2,3]$ and the process shown in Fig. 2 (left).

Rule Based Reasoning. This functionality provides preliminary process design based on experts' experience implemented in terms of 'rules'. The design sequence implemented with the rule based reasoning approach reproduces the way a process designer follows when designing a process: starting from process requirements the tool provides rule based feedback about the basic process design characteristics. This functionality is based on the tool KnowWE [4,5], for which several plug-ins have been developed in order to connect it to KR. A basic implementation is shown in Fig. 2 (right), where the user introduces Material and Constraints (mechanical properties required) and the tool provides process parameters intervals based on implemented rules, in this way defining a preliminary process design, matching user requirements, for optimisation.
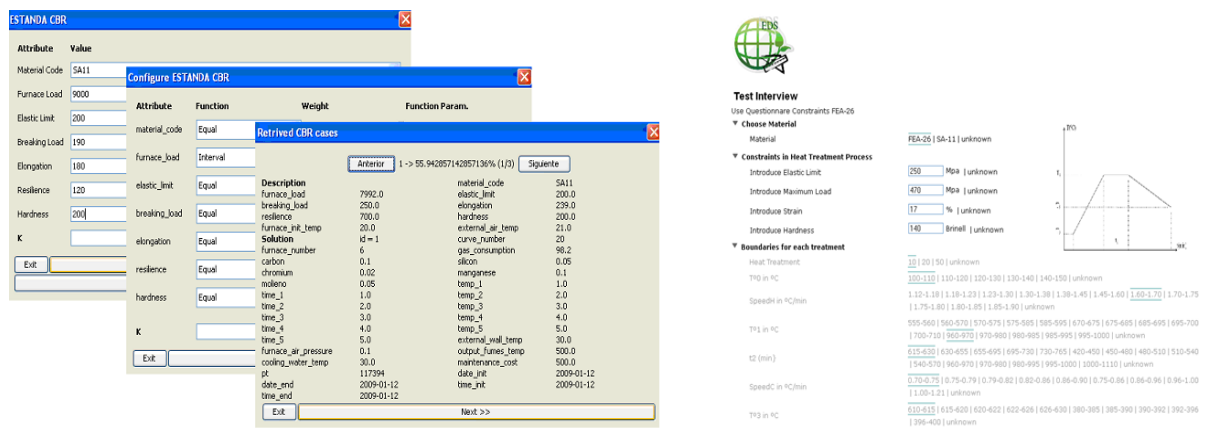

Fig. 2. CBR (left) and RBR (right) implementation for preliminary process design

\subsection{Process Optimisation}

Once a preliminary process design is done with EDS component, Energy Analyser (EA) component provides process optimisation in terms of energy efficiency and/or maintenance cost, keeping required mechanical properties as constraints. EA provides optimal process design, using in turn Energy Simulator (ES) for energy consumption estimation of each potentially optimal design configuration considered.

Process Optimisation with EA. DEMI component EA provides the energy optimised process design from the preliminary design provided by EDS, finding minimum energy given ranges on process variables and constraints on requirements variables. Process variables have to be within their given boundaries and constrained by requirements within constraints given by the user. So EA requires information related to the preliminary process definition (provided by EDS) and boundary limits for all the relevant parameters values. 
The procedure is basically as follows: EA Client calls EA with the process configuration ID; EA reads configuration ID with info about the nodes from the Knowledge Repository (KR), as well as initial data, boundaries for process variables, and constraints for quality variables from KR; EA starts Matlab, as a server, for process optimisation; For energy process optimisation, EA uses function fmincon to find minimum energy given ranges on process variables and constraints on quality variables; EA uses ES as a service to evaluate energy for each iterated process during optimisation, gets energy consumption estimations from ES and is able to predict quality variables (mechanical properties) for each configuration; EA finally sends to KR the process parameters values describing the optimal process design.

Process Energy Consumption Simulation. Energy Simulator (ES) estimates the energy consumption of each possible specific process configuration provided by EA component, so that the minimum energy consumption process configuration can be finally selected.

In general, ES is a tool intended for simulation of network system or process, when energy consumption is of interest. ES is constructed as a simulation service able to simulate new/innovative network or system designs and estimate energy use for them. The analytical modelling and simulation is based on "block-based modelling" concept rather than on purely "process-based modelling". It means that whole physical process is decomposed into sub-processes represented by independent blocks (one block for one sub-process). Sub-process is process variable (output of block) related to physical behaviour of other variables (inputs of block) with their weighting coefficients (block parameters). Sub-process may be expressed as a process variable or function dependent on other process variables [6].

ES requires a theoretical model for energy use estimation provided a process configuration. In the case of heat treatment process at Fundiciones del Estanda a basic thermodynamic model has been considered. This model expresses fuel (gas) consumption and energy balance (see an example in Fig. 3) of the heat treatment process, taking into consideration the following energy terms: Energy provided by the gas, Energy lost by fumes, Heat stored by parts after treatment, Heat stored by furnace refractory, Heat losses through the furnace walls.

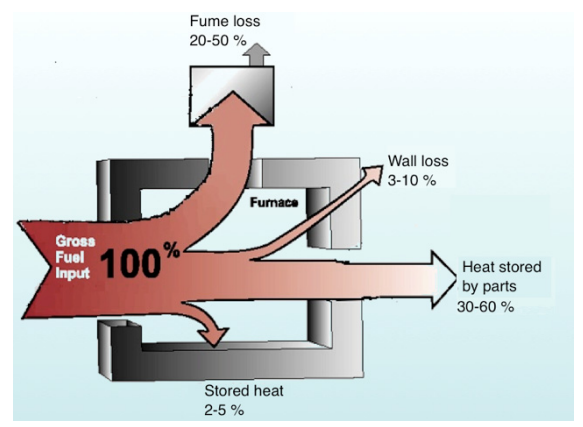

Fig. 3. Example of fuel consumption and energy balance of the heat treatment process 
This theoretical model is the basis of ES component, also running in Matlab, which receives a given process design from EA. The data needed for this energy consumption estimation are the following, given by the process parameters values describing the process: Material type (specific heat) of the treated parts, Parts load (mass) to be treated, Difference between parts final and initial temperature $\left(T_{f i n}-T_{i n}\right)$, Total process time $\left(t_{f i n}-t_{i n}\right)=\Sigma t_{i}$, Configuration of heat treatment process.

Energy balance equation of such a process can be simplified as follows:

$$
Q_{g a s} P_{C I}=M_{F} h_{F}+M_{s t} c_{s t}\left(T_{f i n}-T_{i n}\right)+M_{r e f r} c_{r e f r}\left(T_{r e f-f i n}-T_{r e f-i n}\right)+P_{W} A_{W}\left(t_{f i n}-t_{i n}\right)
$$

These terms, considered along the whole process time, have the following meaning:

- Energy provided by the gas, depending on gas consumption (time dependent) and gas calorific power. Total gas consumption is the parameter to be estimated.

- Energy lost by fumes, depending on fumes mass and enthalpy.

- Heat stored by parts after treatment, depending on the parts load, steel specific heat and temperature net increment of the parts.

- Heat stored by furnace refractory, depending on the furnace refractory mass, its specific heat and temperature net increment.

- Heat losses through the furnace walls, depending on energy loss per unit time (time dependent), furnace walls area and process duration.

In order to structure the workflow of power consumption estimation, ES employs four main functionalities: Get configuration (after unique ID from EA is received, ES will connect via web services to the $\mathrm{KR}$ and retrieve information about the process configuration), Add blocks (identifies different phases of heat treatment process), Specify connections (creates the heating model from specific phases identified in the previous functionality), Calculate energy use of process configuration (performs estimation of used gas volume together with consumed power).

\subsection{Mathematical Modeling Approach for Process Optimisation}

As described above, in order to optimise the heat treatment process design keeping the resulting steel parts' mechanical properties, some mathematical models have to be ready relating the different sets of parameters involved. If an analytical model considering the physics involved is not achievable due to the problem complexity, an empirical model approach should be followed, which would require a huge historic database allowing correlations among parameters be found.

The different sets of parameters considered are the following:

- Heat treatment process parameters, including: Date/time, Material Load, Type of material and Heat treatment Temperature-time curve, for a simplified process defined by initial temperature, maximum temperature, time at maximum temperature and heating/cooling speed.

- Measured resulting mechanical properties of parts after heat treatment processes, currently stored in quality tests documentation at the company. Mechanical 
properties considered are the following: Yield and Ultimate strength, Strain, Resilience and Hardness.

- Maintenance cost parameters of historic processes, currently stored at Fundiciones del Estanda by PRISMA software database, provided by the company Sisteplant [7]. The main maintenance cost parameters considered are: Mean Time Between Failures (MTBF), Number of breakdowns (per year), Cost of breakdown (average working hours, material, non productive time).

In order to optimise the process design these sets of parameters have to be linked through mathematical models and expressed as objective function. It is important to remark that these models are intended to allow a comparison between different process designs performance, rather than accurate predictions on energy use, maintenance cost or mechanical properties, so that alternative process designs proposed by EA can be simulated by ES, compared by EA and finally an improved process design provided as result part of the optimisation procedure. The relations between sets of parameters and mathematical modelling approach considered are the following:

- Relationship between process design parameters and mechanical properties required. This model is based on experts' considerations about the influence of process parameters over the resulting mechanical properties. In this way a process optimisation can be performed while keeping constraints on mechanical properties. For example, optimal process might have a lower heating speed or maximum temperature, in this way decreasing the energy consumption, while resulting mechanical properties are still kept within specifications.

- Relationship between process design parameters and maintenance cost. According to experts' criteria, the impact of process parameters modifications in maintenance cost can be estimated, and optimisation in terms of maintenance cost performed.

- Relationship between process design parameters and energy consumption. A simplified analytical model has been created, as described in section 3.2, allowing an estimation of energy consumption given a process configuration.

\section{Conclusions}

The set of tools developed and here described allows a manufacturing company dealing with heat treatment to optimise the process in terms of energy efficiency and maintenance costs while keeping the mechanical properties of the treated parts requested by the customer. Tools supporting knowledge based decision making as those described in Energy Dependency Selector component allow the company to keep and reuse the experts' knowledge and experience for a quick preliminary process design, while Energy Analyser and Energy Simulator components support the process design optimisation. All these components can work in a distributed way with a common Knowledge Repository, based on web services.

On the other hand, this set of tools can be adapted to other manufacturing processes where correlations between process parameters and resulting quality 
parameters can be established, as well as a model for energy consumption estimation, so the approach and tools here presented are portable to other manufacturing processes and therefore to other manufacturing companies.

Acknowledgements. The work here described is being developed in the context of project DEMI "Product and Process Design for AmI Supported Energy Efficient Manufacturing Installations" (2010-2013), funded by the European Commission under the FP7 (ICT-2009-247831) [8].

\section{References}

1. Friden, H., Bergfors, L., Bjork, A., Mazharsolook, E.: Energy and LCC Optimised Design of Compressed Air Systems: A Mixed Integer Optimisation Approach with General Applicability. In: 2012 UKSim 14th International Conference on Computer Modelling and Simulation, pp. 491-496 (2012)

2. Díaz-Agudo, B., González-Calero, P.A., Recio-García, J.A.: JColibri Tutorial. Facultad de Informatica, Universidad Complutense de Madrid, Spain, http://gaia.fdi.ucm.es/ files/people/juanan/jcolibri/downloads/tutorial.pdf

3. Díaz-Agudo, B., González-Calero, P.A., Recio-García, J.A.: Building CBR systems with Jcolibri. Science of Computer Programming 69(1-3), 68-75 (2007)

4. Baumeister, J., Reutelshoefer, J., Puppe, F.: KnowWE - Community based Knowledge Capture with Knowledge Wikis. In: K-CAP 2007: Proceedings of the 4th International Conference on Knowledge Capture, pp. 189-190 (2007)

5. Baumeister, J., Reutelshoefer, J., Puppe, F.: Web-Based Knowledge Engineering with KnowledgeWikis. In: Proc. of AAAI 2008 Spring Symposium on Symbiotic Relationships between Semantic Web and Knowledge Engineering, pp. 1-13. Stanford University, USA (2008)

6. Iešmantas, T., Alzbutas, R.: Designing of energy efficient system considering power consumption and reliability. In: 9th Annual Conference of Young Scientists on Energy Issues CYSENI 2012: International Conference, May 24-25, pp. 179-189. LEI, Kaunas (2012) ISSN 1822-7554

7. http://prisma.sisteplant.com/

8. http://www. demi-online.eu/ 